The method for direct excitation is the following:- The excitor of the pharynx is inserted in the pharynx as low down as the posterior and inferior part of the larynx. The second wet excitor being placed outside, in the level of the crico-thyroid muscle, the former is conducted on the posterior surface of the larynx downwards and upwards, and vice versic. Indirect Faradization of the larynx is much easier:- The excitor is directed on the sides of the inferior constrictor to reach the inferior laryngeal nerve, animating all the inner muscles of the larynx; whilst the other wet excitor is placed on the nape of the neck.

Direct Faradization of the heart and the lungs, the stomach and the liver, is not possible; but stomach, lungs, and heart may be excited indirectly by Faradization of the tenth pair, accessible through the pharynx and osophagus. These operations seem, however, to be dangerous and not useful, so that a conscientious physician will seldom, if ever, consent to employ them.

I conclude with a few words about the electric excitation of the diaphragm, easily to be produced by Faradization of the phrenic nerve, which, taking its rise from the third, fourth, and fifth cervical pairs, proceeds downwards and inwards in front of the scalenns anticus, before it reaches the mediastinum and the diaphragm. On the anterior surface of the scalenus anticus the phrenic nerve may be easily excited. Excitors with large surfaces, viz., sponges thrust in metallic cylinders, are kept on the point alluded to, and instantly the artificial respiration is produced; the thorax is expanded, and the air rushes into the lungs with considerable noise. So it is possible to maintain respiration in a body even some time after death; and it may be easily conceived how important this agent may become in asphyxia, whether it be produced by charcoal fumes, by opium, by chloroform, or by cholera. In all these cases the first indication is, to induce respiration, which is often to save life.

Manchester-street, July, 1857.

\section{REPORT OF A CASE OF ASIATIC CHOLERA.} BY HENRY MADGE, M.D.

Os Wednesday, Aug. 12th, I was called to a gentleman in Gower-street, who had the following symptoms:-Complete prostration, speaking in a. whisper; violent cramps, particnlarly in the feet, legs, and abdomen; finger-nails purple, and the skin of the fingers shrivelled and cold, like those of a dead subject; the arms, legs, and feet were also quite cold; the eyes were dimmed and sunken, and, as there was labouring for breath, the face wore a most anxious expression; pulse feeble and irregular, with sickness and profuse evacuations of ricewater stools. The patient was evidently sinking from an attack of real Asiatic cholera. I immediately ordered hot water to the feet, warm flannels to the legs and arms, placed a large mustard poultice over the whole abdominal region, administered stimulants, and prescribed the following mixture:Acetate of lead, fifteen grains; acetic acid, half a drachm; tincture of opium, half a drachm; tincture of capsicum, eight minims; and peppermint-water to six ounces: two tablespoonfuls to be taken immediately.

On calling again, in an hour, I met Mr. Weekes, of Bloomsbury, the regular medical attendant, and we had the satisfaction of finding that the mixture had kept down, and that, although previously the evacuations had been constant, they appeared now to have ceased. The other symptoms had undergone no material change: the patient's whispers were scarcely audible, tongue dry and furred, urine high-coloured, and in small dribbling quantities. The mixture was ordered to be taken every hour; we also ordered iced water to allay thirst, port-wine, and the constant application of warmth to the extremities. In the evening, reaction had commenced; pulse quick, but still weak and irregular; returning warmth; slight. sickness, but no diarrhoea; cramps in feet and legs still severe, The above treatment was persevered in during the night.

The next day, August 13 th, we found reaction perfectly established; voice still husky and indistinct; cramps in legs troublesome; pulse improved, tongue furred and accompanied with considerable thirst. Mixture to be taken less frequently, and iced water more freely. Late in the day a copious dark watery stool came away, having the appearance of discoloured rice-water, which may probably have been owing to the action of disengaged gas on the lead taken into the system. Mixture to be taken at shorter intervals.

Aug. 14th. - Has had a good night; perspired profusely; no diarrhoea ; tongue still coated, but moist; pulse almost natural; passed a little more urine; ominous rumbling sounds in the intestinal canal, but without pain. Ordered beef-tea and moderate quantities of port-wine with arrowroot; a dose of the mixture at night, to be repeated, if necessary.

15th. - Improved in every respect; bowels have not acted. To discontinue the mixture. Ordered mercury with chalk at bed-time.

16th. - Continues to improve. Bowels not having acted, ordered castor oil.

17th.-Quite convalescent; feels weak, but able to get up and walk about. The castor oil has acted three times; stools dark, bilious, and partially firm; appetite returning. Ordered quinine mixture, and a lit tle carefully.got-up meat diet.

As the object of pablishing cases of this kind is to draw from them practical deductions, I should be glad to be permitted to offer one or two remarks. The mixture $I$ employed in this case, and which acted so admirably, was recommended to me by a practitioner in 1854 . I then held an appointment under the Local Board of Health, and besides several hundred cases of diarrhcea and choleraic diarrhcea, I treated several severe cases of genuine cholera. Six of the number terminated. fatally, but in the majority of them I proved the efficacy of the means employed in this case. There was one extreme case, at the bottom of Rathbono-place, which two medical men and others will well remember yielded to the same treatment; not, however, before I was fortunate enough to sablue a violent sickness by giving creosote. As an astringent, the plumbi acetas seems to act quicker and better in solution than in pills, and it is an advantage to be able to combine with it tho tincture of capsicum, which certainly seems to stimulate and com. fort the patient, whilst it assists the opium in relieving painfin spasms. In 1839 I attcnded cases of cholera at Bow, and was in the habit of giving calomel. After that fearful visitation had passed away, on reviewing my cases, I entertained the opinion that calomel was injurious: my subsequent experience has only tended to confirm this opinion. To me, it has appeared to add to the depression of the patient at a time when he requires to be supported and even stimulated; and from its well-known effects in other cases, it must necessarily keep up the excessive irritation in the mucous cont of the intestines, and the consequent serous effusion which helps to kill the patient. In the opportunities I have had (many of them at Guy's) of seeing post-mortem examinations in cases of cholera, the gall-bladder has invariably been found distended with bile. It appears to have been prevented from emptying itself into the usual channel by a spasmodic closure of its neck, or from the ductus communis and its connexions partaking of the general spasm affecting the system. If this is the case, what good can calomel do? The liver does not want stimulatingthere is already an abundance of bile. Is it not better, in collapse, and in the dangerous stages of the disease, to trust to stimulants, astringents, and antispasmodics? The latter will bring about what is supposed to be the effect of mercury in such cases-the passage of bile. When the severe symptoms are over, the internal organs having passed through a state of congestion, a mild mercurial appears to be advisable, and, as in the foregoing case, will probably act well. Of course there will be differences of opinion on these views; but the case I have related will serve to show, first, that our old enemy the cholera is amongst us; and secondly, that in a very severe form, in the stage of collapse, it has been met and treated successfully.

Howland-street, Fitzroy-square, Aug. 1857.

\section{SECOND QUARTERLY METEOROLOGICAL} REPORT AT ST. THOMAS'S HOSPITAL FOR 1857.

BY ROBERT DUNDAS THOMSON, M.D., F.R.S. L. \&.E., PROFESSOR OF CHEMISTIX IN ST. THOMAS'S HOSPITAL COLLEGE, EXAMINER IN CHEMISTRY AT THE UNIVEISITY OF LONDON, ETC.

THE temperature of April was two degrees lower than that of the corresponding month of last year, but was only 6 degree inferior to the mean for London during 65 years. May was five degrees hotter than the same month in 1856, while 